Website: http://revistas.lamolina.edu.pe/index.php/acu/index

(C) Universidad Nacional Agraria La Molina, Lima - Perú

\title{
Insumos orgánicos en la preparación de sustratos para el crecimiento de Dianthus chinensis y Fuchsia sp.
}

\author{
Organic Materials In Substrate Conditioning For Growing to Dianthus chinensis y Fuchsia sp.
}

Juan Carlos Jaulis C. ${ }^{1}$; Alejandro Pacheco A. ${ }^{2}$; A. Martinez V. ${ }^{3}$; Sarita Moreno Ll. ${ }^{4}$

\begin{abstract}
Resumen
El objetivo de la presente investigación fue evaluar el crecimiento y desarrollo de Dianthus chinensis y Fuchsia sp. en contenedores cuya composición de sustratos son insumos orgánicos. Los tratamientos evaluados fueron: tierra de chacraaserrín 1:1 (T1), tierra de chacra-compost 1:1(T2), tierra de chacra-musgo 1:1(T3), compost-aserrín 1:1(T4), compostmusgo 1:1(T5), estiércol de caballo-aserrín 1:1(T6) y estiércol de caballo-musgo 1:1(T7). Se usó un diseño de bloques completos al azar (DBCA), siete tratamientos, tres repeticiones, ocho unidades en cada tratamiento, para cada especie evaluada. Para la comparación de medias se utilizó la prueba estadística de Duncan a un nivel de significancia de 0,05 . Se utilizaron clavelinas de 30 días, con dos hojas, cuya propagación fue por semilla botánica, mientras que la Fuschia utilizadas tenían tres pares de hojas, ocho centímetros de altura y fueron propagadas por esqueje, ambas bajo condiciones de invernadero. El mejor tratamiento fue el T5, que ofreció las mejores propiedades físicas y químicas para el buen crecimiento y desarrollo de Dianthus chinensis (clavelina) y Fuchsia sp. (Fuchsia) en las variables de crecimiento, biomasa y rendimiento evaluadas. En ambos cultivos los T1, T4 y T6 tuvieron menores rendimientos en las variables evaluadas debido a la presencia de sales, en concentraciones perjudiciales para el crecimiento y desarrollo del cultivo, que contiene los insumos tierra de chacra, compost y estiércol de caballo.
\end{abstract}

Palabras clave: Dianthus chinensis; Fuchsia sp.; musgo; aserrín.

\begin{abstract}
The objective of the present investigation was to evaluate the growth and development of Dianthus chinensis and Fuchsia sp. in containers whose composition of substrates are organic inputs. The treatments were evaluated: earth of chacra-sawdust 1: 1 (T1), land of chacra-compost 1: 1 (T2), earth of chacra-moss 1: 1 (T3), compost-sawdust 1: 1 (T4), compost-moss 1: 1 (T5), horse manure-sawdust 1: 1 (T6) and horse-moss manure 1: 1 (T7). A randomized complete block design (DBCA) was used, seven treatments, with three repetitions, eight units in each treatment, for each evaluated species. For the comparison of means, Duncan's statistical test was used at a significance level of 0,05. Clavelins were used for 30 days and with two leaves, whose propagation was by botanical seed, while the Fuschia used had three pairs of leaves, eight centimeters high and were propagated by cutting, both under greenhouse conditions. The best treatment was T5, which offered the best physical and chemical properties for the good growth and development of Dianthus chinensis (Clavelina) and Fuchsia sp. (fuchsia) in the variables of growth, biomass and yield evaluated. In both crops, T1, T4 and T6 had lower yields in the evaluated variables due to the presence of salts, in concentrations that are detrimental to the growth and development of the crop, which contains the inputs of farmland, compost and horse manure.
\end{abstract}

Keywords: Dianthus chinensis; Fuchsia sp.; moss; sawdust.

\section{Introducción}

En los últimos años el cultivo de plantas ornamentales ha tomado mayor importancia en el Perú (Jaulis y Pacheco, 2015), debido al interés por el cuidado y conservación del medio ambiente, que conlleva a mejorar la calidad de vida de la población. La OMS (Organización Mundial de la Salud) recomienda $9 \mathrm{~m}^{2}$ de áreas verde/habitante para una buena calidad de vida (RPP, 2017). Sin embargo, a setiembre del 2016 Lima contó con $2,71 \mathrm{~m}^{2}$ de área verde/ habitante, valor inferior a lo recomendado por la OMS
(Instituto Metropolitano de Planificación, 2012). Por ello, las autoridades públicas han dispuesto políticas para la creación e implementación de espacios con vegetación, donde las personas se recreen de manera segura y saludable; incrementado la producción de plantas ornamentales en los viveros públicos y privados con el fin de atender la creciente demanda (Armijos y Barrezueta, 2016).

Los cultivos intensivos de plantas ornamentales han impulsado la producción de flores en contenedores, con diversos materiales, conocidos como sustratos, que

\footnotetext{
${ }^{1}$ Departamento de Horticultura, Facultad de Agronomía, Universidad Nacional Agraria La Molina, Lima, Perú. E-mail: jjaulis@lamolina.edu.pe ${ }^{2}$ Departamento de Horticultura, Facultad de Agronomía, Universidad Nacional Agraria La Molina, Lima, Perú. Email: aari@lamolina.edu.pe

${ }^{3}$ Investigador independiente, Lima, Perú. Email: marva0781@hotmail.com

${ }^{4}$ Departamento de Horticultura, Facultad de Agronomía, Universidad Nacional Agraria La Molina, Lima, Perú. Email: saritamoreno@lamolina.edu.pe.
} 
desplazaron la producción tradicional del cultivo en suelo. Un sustrato es el material sólido natural, de síntesis o residual, orgánico o mineral, puro o mezclado (FortisHernández et al., 2012) que en un contenedor permite el anclaje del sistema radical, da soporte a la planta e interviene o no en su nutrición (Avenza, 2018). Los sustratos se clasifican en inertes, si solo brindan soporte a la planta, y activos, si proporcionan además nutrimentos (Pastor, 2000; Abad et al., 2005). Los estudios de sustratos señalaban que la obtención de plantas y flores con calidad alta dependía en gran parte de las características físicas y químicas del sustrato (Ansorena, 1994; Cabrera, 1999).

El cultivo industrial de plantas ornamentales comprende una serie de prácticas culturales, donde la selección y el manejo de los sustratos influye en la calidad de la planta (Valenzuela et al., 2014). La importancia de la selección y la correcta manipulación de los insumos para el sustrato reside en las funciones básicas que debe cumplir como proveer agua, permitir el intercambio gaseoso hacia las raíces, ser reservorio de nutrimentos y brindar soporte físico para las plantas (Handreck y Black, 2002). También, debe ser amortiguador de los cambios ambientales bruscos que podrían afectar el crecimiento de los cultivos (Pérez et al., 2016). Además, debe cumplir con características físicas (elevada capacidad de retención de agua fácilmente disponible; suficiente suministro de aire; buena distribución de tamaño de partículas; baja densidad aparente; elevada porosidad total; estructura estable y contracción reducida) y químicas (baja a moderada CIC, balance de nutrientes asimilables, baja salinidad, $\mathrm{pH}$ moderadamente ácido, elevada capacidad tampón, mínima velocidad de descomposición) adecuadas que representan una alternativa para sustituir sustratos comerciales de alto costo e impacto ambiental negativo (Gayosso-Rodríguez et al., 2018). Adicionalmente, Abad et al. (2005) reportaron que debe ser fácil de mezclar; humectar, desinfectar, estar libre de semillas de malezas; nematodos y otros patógenos. Por ello, en las últimas décadas, en Latinoamérica se evaluaron diversos sustratos orgánicos que son ecológicos, económicos y de disponibilidad local (Gayosso et al., 2016). Una opción según Valenzuela et al. (2014) son los residuos, subproductos agroindustriales y materiales subvalorados que, al ser incorporados a los sistemas productivos agrícolas como sustratos, se convierten en una alternativa para la producción intensiva.

El musgo es un sustrato (Huarcaya, 2014), cuyo precio cada año incrementa, su extracción genera un impacto ambiental negativo (Eltelégrafo, 2017), por ser cosechado desde la raíz, dejando el suelo desnudo de las zonas altoandinas que, en época de lluvia, diciembre a marzo, generará erosión hidrica. Por lo tanto, ante la alta demanda de musgo, se tienen otras alternativas como: estiércol de caballo, cascarilla de arroz, compost, cascarilla de café, aserrín, entre otros insumos, que al mezclarlos en proporciones adecuadas pueden servir como sustrato (VIFINEX, 2002).
El aserrín, es un material localmente disponible, producto de los aserraderos (Juárez et al., 2001), muy utilizado en viveros pequeños y su uso como sustrato es recomendado por diversos autores (Barzegar et al., 2015; Pineda-Pineda et al., 2012), por los buenos resultados en la producción de plantas de diferentes especies (Burés, 1997; Hartmann y Kester, 1998), como Capsicum annum L. (Barzegar et al., 2015), Solanum lycopersicum L. (Vargas et al., 2014) entre otros. Los resultados indicaron que su utilización como sustrato es una alternativa para el cultivo de plantas en contenedor (Gayosso-Rodríguez et al., 2018). Sin embargo, presenta algunas desventajas como una alta relación carbono-nitrógeno, liberación de sustancias fitotóxicas que pueden inhibir o dañar la formación y crecimiento del sistema radicular afectando el desarrollo del cultivo.

Por otra parte, el compost es producto de la degradación de residuos orgánicos (Oviedo-Ocaña, 2012) puede utilizarse como sustituto de otros elementos orgánicos como la turba, contiene nutrientes, mejora la estructura del suelo, la fertilidad y el crecimiento de las plantas; de esta manera, se contribuye con la sostenibilidad de la producción agrícola y con la mitigación del impacto generado por el manejo del residuo (Zurbrügg et al., 2005). Mientras que, según Jardinería (2016) el estiércol de caballo, es uno de los mejores abonos para que las plantas crezcan sanas y fuertes, por el aporte de nutrientes al suelo (Palacios, 2007), el incremento en la retención de humedad y por el incremento de la actividad biológica (Yagodín, 1986).

Por lo anterior, el presente trabajo tuvo como objetivo evaluar el crecimiento y desarrollo de Dianthus chinensis y Fuchsia sp. con diferentes insumos orgánicos (musgo, aserrín, estiércol de caballo, compost) en la preparación de sustratos para contenedores.

\section{Materiales y métodos}

La presente investigación se desarrolló durante siete meses, desde octubre a mayo, en el invernadero del Programa de Investigación en Plantas Ornamentales de la Universidad Nacional Agraria La Molina (UNALM), Lima, Perú. Ubicada geográficamente a una latitud de $12^{\circ} 06^{\prime} \mathrm{S}$, longitud $76^{\circ} 57^{\prime} \mathrm{O}$ y altitud 243,7 m.s.n.m. Ubicado dentro un desierto subtropical árido caluroso según el sistema modificado de Koppen (Garcia, 2004), con temperatura media anual de $18,5^{\circ} \mathrm{C}$, radiación anual de 186,5 cal-g* $\mathrm{cm}^{-1 *}$ día $^{-1}$, humedad relativa anual de $85 \%$ en peso y precipitación anual acumulada de $6 \mathrm{~mm}$.

Los insumos utilizados fueron: compost, musgo, tierra de chacra, aserrín y estiércol de caballo, que son comúnmente utilizados en la producción de plantas ornamentales en el Perú. Previo a su utilización, se realizó un análisis de caracterización en el Laboratorio de Análisis de Suelos, Plantas, Aguas y Fertilizantes (LASPAF) de la facultad de Agronomía de la UNALM (Tabla 1). 
Tabla 1. Características químicas y físicas de los insumos utilizados para la preparación de sustratos

\begin{tabular}{|c|c|c|c|c|c|c|c|}
\hline Insumos & $\mathrm{pH}$ & $\mathrm{CE}(\mathrm{dS} / \mathrm{m})$ & M.O. (\%) & $\begin{array}{c}\text { CIC } \\
(\mathrm{meq} / 100 \mathrm{~g})\end{array}$ & $\begin{array}{l}\mathrm{Hd} \\
(\%)\end{array}$ & $\begin{array}{c}\text { Clase } \\
\text { textural }\end{array}$ & $\begin{array}{c}\mathrm{DP} \\
(\mathrm{mm})\end{array}$ \\
\hline Aserrín & 7,46 & 1,67 & 80,90 & 22,00 & 45,04 & & 0,5 \\
\hline Compost & 7,29 & 4,69 & 29,17 & 36,16 & 29,98 & & 6 \\
\hline Musgo & 6,31 & 0,69 & 53,13 & 20,80 & 73,60 & & 20 \\
\hline Estiércol de caballo & 8,41 & 20,70 & 55,45 & 42,00 & 17,20 & & 2 \\
\hline Tierra de chacra & 7,27 & 3,63 & 1,09 & 12,16 & & Franco & \\
\hline
\end{tabular}

Fuente: Laboratorio de Análisis de Suelos, Plantas, Aguas y Fertilizantes del departamento de Agronomía de la UNALM (2009).

$\mathrm{CE}=$ conductividad eléctrica, $\mathrm{MO}=$ materia orgánica, $\mathrm{CIC}=$ capacidad de intercambio catiónico. $\mathrm{Hd}=$ humedad, $\mathrm{DP}=\mathrm{diámetro}$ de partícula.

El experimento se condujo bajo un Diseño de Bloques Completos al Azar (DBCA) con tres bloques, siete tratamientos, tres repeticiones, con ocho unidades cada tratamiento, para cada especie evaluada. Para el análisis de las variables evaluadas se realizó el análisis de variancia (ANVA) y la comparación de medias se realizó mediante la prueba estadística de Duncan, con un nivel de significancia de 0,05 .

Los tratamientos fueron: tierra de chacra-aserrín 1:1(T1), tierra de chacra-compost 1:1(T2), tierra de chacramusgo 1:1(T3), compost-aserrín 1:1(T4), compost-musgo 1:1(T5), estiércol de caballo-aserrín 1:1(T6) y estiércol de caballo-musgo 1:1(T7) para cada ensayo.

Las variables que se evaluaron al finalizar el experimento fueron: altura de planta $(\mathrm{cm})$, medida desde el cuello hasta la yema terminal más alta $(\mathrm{cm})$; diámetro de tallo, medido a un $1 \mathrm{~cm}$ del cuello de planta $(\mathrm{cm})$, número de hojas (unidad), número de raíces total por planta (unidad), número de brotes (unidad). El conteo de número de flores se realizó desde la aparición de la primera flor hasta culminar el ensayo (unidad).

La preparación de los sustratos consistió en realizar mezclas con los insumos, en proporciones de 1:1. Los sustratos fueron pesados en bolsas de polipropileno de 10 " x 11" pulgadas de acuerdo a la proporción indicada, se colocaron en distintas bolsas, se realizó la mezcla hasta que todo el sustrato quedó homogéneo.

Las especies ornamentales utilizadas en el desarrollo de la presente investigación fue la clavelina (Dianthus chinensis) y la Fuchsia(Fuchsia sp.), especies ornamentales, una de estación y la otra perenne. Se utilizaron clavelinas que tenían 30 días de edad, dos hojas y cuya propagación fue por semilla botánica, mientras que las Fuschias utilizadas tenían tres pares de hojas, ocho centímetros de altura y fueron propagadas por esqueje, ambas bajo condiciones de invernadero. Se plantó un individuo por contenedor de 10" $\mathrm{x} 11$ ". Los riegos fueron frecuentes y ligeros de acuerdo a las condiciones climáticas, se realizó dos deshierbos para controlar las malezas y se aplicó insecticidas (metomil al $90 \%$ polvo soluble, clorpirofos $278 \mathrm{~g} \mathrm{~L}^{-1}$ y dimetoato $22 \mathrm{~g}$ $\mathrm{L}^{-1}$ ) para prevenir la presencia de plagas.

\section{Resultados y discusión}

\section{Caracterización de sustratos}

En la Tabla 2 se presenta las características químicas y físicas de los sustratos evaluados. Los resultados de $\mathrm{pH}$ obtenidos en la presente investigación, indican que el tratamiento T5 fue el único sustrato que presentó un valor $(7,11)$, valor cercano al óptimo, según lo recomendado por Abad (1993) y el reporte de López-Baltazar et al. (2013), quienes señalan que el rango óptimo de $\mathrm{pH}$ es de 5,2 a 6,3. El resultado obtenido fue producto de la fase termófila de producción de compost, por la liberación de aminas, producto de la degradación de compuestos orgánicos como proteínas, bases nitrogenadas (Tortarolo et al., 2008) estabilizándose el compost. Respecto al musgo (Distichia muscoides) que crece en zonas altoandinas húmedas, presentó un $\mathrm{pH}$ de 6,31 (ligeramente ácido), su naturaleza ácida se debe a las paredes de sus células que atrapan gran cantidad de iones básicos $\left(\mathrm{Ca}^{+}, \mathrm{Mg}^{+}, \mathrm{Na}^{+}\right)$y liberan iones $\mathrm{H}^{+}$, haciendo de su entorno un medio ácido que es adecuado para su crecimiento y desarrollo.

Tabla 2. Características químicas y físicas de los sustratos evaluados

\begin{tabular}{|c|c|c|c|c|c|c|c|c|c|}
\hline Tratamientos & Proporción & $\mathrm{pH}$ & $\begin{array}{c}\mathrm{CE} \\
\mathrm{dS} . \mathrm{m}^{-1} \\
\end{array}$ & $\begin{array}{c}\text { CIC } \\
\text { Meq. } 100 \mathrm{~g}^{-1}\end{array}$ & $\begin{array}{c}\mathrm{MO} \\
\% \\
\end{array}$ & $\begin{array}{c}\text { DA } \\
\text { g.cm }{ }^{-3}\end{array}$ & $\begin{array}{c}\text { DR } \\
\text { g.cm }{ }^{-3}\end{array}$ & $\begin{array}{c}\text { D } \\
\text { g.cm }{ }^{-3}\end{array}$ & $\begin{array}{c}\text { MRH } \\
\% \\
\end{array}$ \\
\hline Tierra de chacra-aserrín & $1: 1$ & 7,33 & 3,04 & 11,20 & 17,21 & 0,65 & 1,88 & & 64,43 \\
\hline Tierra de chacra-compost & $1: 1$ & 7,28 & 5,17 & 18,08 & 8,19 & 0,84 & 2,18 & & 90,65 \\
\hline Tierra de chacra- musgo & $1: 1$ & 7,36 & 3,65 & 11,52 & 1,84 & 1 & 2,18 & & 87,58 \\
\hline Compost-aserrín & $1: 1$ & 7,41 & 3,09 & 32,48 & 45,16 & & & 0,36 & 174,56 \\
\hline Compost-musgo & $1: 1$ & 7,11 & 3,52 & 40,00 & 37,85 & & & 0,21 & 202,45 \\
\hline Est. de caballo-aserrín & $1: 1$ & 8,13 & 11,1 & 30,80 & 65,25 & & & 0,20 & 217,67 \\
\hline Est. de caballo-musgo & $1: 1$ & 8 & 15,7 & 45,60 & 58,79 & & & 0,10 & 301,81 \\
\hline
\end{tabular}

Fuente: Laboratorio de Análisis de Suelos, Plantas, Aguas y Fertilizantes del departamento de Agronomía de la UNALM (2009).

$\mathrm{CE}=$ conductividad eléctrica, $\mathrm{CIC}=$ capacidad de intercambio catiónico, $\mathrm{MO}=$ materia orgánica, $\mathrm{DA}=$ densidad aparente, $\mathrm{DR}=$ densidad real, $\mathrm{D}=$ densidad, MRH= máxima retención de humedad. 


\section{Conductividad eléctrica (CE)}

El Tratamiento T1 presentó la menor C.E. $\left(3,04 \mathrm{dSm}^{-1}\right)$, ello se debió a la composición del sustrato que resultó de la mezcla de tierra de chacra $\left(3,63 \mathrm{dSm}^{-1}\right)$ y aserrín $(1,67$ $\left.\mathrm{dSm}^{-1}\right)$. Asimismo, los Tratamientos 3, 4 y 5 se encuentran dentro del rango deseable de acuerdo con el reporte de Cabrera (1999). Mientras que el T2 presentó una salinidad moderada, característica que se atribuye al estiércol de caballo $\left(20,70 \mathrm{dSm}^{-1}\right)$, materia orgánica utilizada para la elaboración del compost. Sin embargo, al realizar el proceso de compostaje, el lavado disminuyó la cantidad de sales disminuyendo su conductividad eléctrica a 4,69 $\mathrm{dSm}^{-1}$ pero al realizar la mezcla con tierra de chacra la CE fue de $5,17 \mathrm{dSm}^{-1}$.

\section{Capacidad de intercambio catiónico (CIC)}

Los resultados de la investigación (Tabla 2) indican que los sustratos compuestos por insumos orgánicos presentan mayor CIC (mayor a 20 meq100g ${ }^{-1}$ ) en comparación de los compuestos por insumos inorgánicos (tierra de chacra, con $\mathrm{CIC}=12,16$ meq $100 \mathrm{~g}^{-1}$ ), concordando con Burés (1998); Handreck y Black (2002) quienes indican que los materiales orgánicos presentan cuatro a cinco veces más CIC que los compuestos de materiales minerales. Dicho resultado se debe porque en los suelos la reactividad está dada por el área superficial y la carga de la superficie es producto del proceso de sustitución isomórfica e ionización de grupos funcionales, siendo influenciados por el factor $\mathrm{pH}$ (Mengel y Kirkby, 2004).

\section{Materia orgánica (MO)}

En la Tabla 2 se presenta el porcentaje de MO de los sustratos, observándose diferencias entre tratamientos debido a la composición de los insumos. El T6 presentó el mayor porcentaje de MO (65,25\%), seguido del T7, con $58,79 \%$; T4, con $45,16 \%$ y el T5, con $37,85 \%$. Mientras que los T1, T2 y T3 presentaron el menor porcentaje de materia orgánica debido que contenían tierra de chacra.

\section{Densidad aparente y real}

De los siete tratamientos, el T3 presentó la mayor densidad aparente $\left(1 \mathrm{~g} \mathrm{~cm}^{-3}\right)$, mientras que el T1 y T2 presentaron la menor densidad aparente, con $0,65 \mathrm{~g} \mathrm{~cm}^{-3}$ y $0,84 \mathrm{~g}$ $\mathrm{cm}^{-3}$ respectivamente (Tabla 2), resultado mayor a lo recomendado por Abad y Noguera (2000) para el cultivo de plantas en contenedor en invernaderos $\left(0,15 \mathrm{~g} \mathrm{~cm}^{-3}\right)$, Baudoin et al. (2002) recomienda $0,22 \mathrm{~g} \mathrm{~cm}^{-3}$ mientras que Quintero et al. (2011) reportaron 0,50 a $0,75 \mathrm{~g} \mathrm{~cm}^{-}$ ${ }^{3}$. Asimismo, es importante determinar la porosidad del sustrato, propiedad física de gran importante en horticultura ornamental (Iskander, 2002).

De los tratamientos, el T1 presentó el valor más bajo $\left(1,88 \mathrm{~g} \mathrm{~cm}^{-3}\right)$ mientras que el T2 y T3 presentaron valores iguales, con una densidad real de $2,18 \mathrm{~g} \mathrm{~cm}^{-3}$, encontrándose dentro del nivel óptimo recomendado $(1,45-2,65 \mathrm{~g} \mathrm{~cm}$ $\left.{ }^{3}\right)$ por Abad (1993) para sustratos. Esta propiedad física está determinada por la composición de las partículas que constituyen el sustrato, teniendo en todos los casos un $50 \%$ de materia orgánica (aserrín, compost y musgo) ocasionando que sus valores sean relativamente bajos.

\section{Densidad}

En la Tabla 2 se observa que el T4 presenta la mayor densidad $\left(0,36 \mathrm{~g} \mathrm{~cm}^{-3}\right)$ seguido del T5 $\left(0,21 \mathrm{~g} \mathrm{~cm}^{-3}\right)$, T6 $\left(0,2 \mathrm{~g} / \mathrm{cm}^{3}\right)$ y T7 $\left(0,1 \mathrm{gcm}^{-3}\right)$. Los resultados obtenidos se debieron a los insumos orgánicos que fueron utilizados en la preparación del sustrato. La materia orgánica se descompone y mineraliza para ser absorbido por el cultivo, disminuyendo el volumen del sustrato presente en el contenedor, alterando significativamente las propiedades físicas en el tiempo. Para Mascarini et al. (2012) la densidad del sustrato depende del porcentaje de porosidad, esta característica afecta directamente la velocidad de filtración del agua y la retención de la humedad, siendo la característica física más significativa para la horticultura ornamental en contenedor. Sin embargo, es importante que un porcentaje de los poros no estén cubiertos por agua, para permitir la oxigenación de las raíces y el intercambio de gases entre la atmósfera y sustrato; para lo cual Morales y Casanova (2015) sugieren que el sustrato debe contener entre 10 a $30 \%$ de aire.

\section{Máxima retención de humedad}

Los T1, T2 y T3 presentaron menores valores de retención de humedad con 64,$43 ; 90,65$ y $87,58 \%$ respectivamente, en comparación con los T4, T5, T6 y T7 que presentan $174,56 \% ; 202,45 ; 217,67$ y $307,81 \%$ respectivamente. Los mayores porcentajes de retención lo obtuvieron los sustratos conformados por insumos orgánicos. Sin embargo, Morales y Casanova (2015) reportaron que la mezcla de materiales orgánicos con inorgánicos, con partículas mayores a $1 \mathrm{~mm}$ respecto al orgánico, favorecen la formación de poros que a su vez favorecen la retención de humedad. Por ello, Ansorena (1994) y Urrestarazu (2015) indican la necesidad de considerar el porcentaje de retención de humedad al seleccionar el sustrato.

\section{Clavelina (Dianthus chinensis)}

En la Tabla 3 se muestra los resultados de las variables evaluadas en el Dianthus chinensis que fueron: altura de planta (Ap), número de hojas por planta $\left(\mathrm{N}^{\circ} \mathrm{h}\right)$, número de brotes por planta $\left(\mathrm{N}^{\circ} \mathrm{b}\right)$, diámetro de tallo por planta $(\mathrm{Dt})$, número de raíces por planta $\left(\mathrm{N}^{\circ} \mathrm{r}\right)$ y número de flores por planta $\left(\mathrm{N}^{\circ} \mathrm{f}\right)$. El T5 mostró la mejor respuesta en todas las variables evaluadas en comparación con los demás tratamientos. En relación a la altura todos los tratamientos a excepción del T4 alcanzaron una altura que se encuentra entre 15 a $20 \mathrm{~cm}$, rango reportado por Sakata Seed Sudamerica ltda (2011), similar resultado reportó Oberpaur et al. (2010) al cultivar lechuga en sustratos compuestos por mezclas de musgo-humus (60-40\%) y musgo-compost (60-40 \%), siendo estas proporciones parecidas a la que utilizamos en el cultivo de clavelina. 
Tabla 3. Variables evaluadas en Dianthus chinensis en diferentes sustratos

\begin{tabular}{lccccccc}
\hline \multicolumn{1}{c}{ Tratamientos } & T & $\begin{array}{c}\mathrm{Ap} \\
\text { (cm) }\end{array}$ & $\begin{array}{c}\mathrm{N}^{\circ} \mathrm{h} \\
\text { (und) }\end{array}$ & $\begin{array}{c}\mathrm{N}^{\circ} \mathrm{b} \\
\text { (und) }\end{array}$ & $\begin{array}{c}\mathrm{D} \mathrm{t} \\
\text { (cm) }\end{array}$ & $\begin{array}{c}\mathrm{N}^{\circ} \mathrm{r} \\
\text { (und) }\end{array}$ & $\begin{array}{c}\mathrm{N}^{\circ} \mathrm{f} \\
\text { (und) }\end{array}$ \\
\hline Tierra de chacra- aserrín & $\mathrm{T} 1$ & $16,27 \mathrm{abc}$ & $19,66 \mathrm{~d}$ & $2,91 \mathrm{~cd}$ & $0,24 \mathrm{~d}$ & $5,11 \mathrm{e}$ & $07,33 \mathrm{e}$ \\
Tierra de chacra-compost & $\mathrm{T} 2$ & $17,63 \mathrm{ab}$ & $69,50 \mathrm{~b}$ & $3,83 \mathrm{~b}$ & $0,34 \mathrm{~b}$ & $9,22 \mathrm{~b}$ & $48,58 \mathrm{ab}$ \\
Tierra de chacra-musgo & $\mathrm{T} 3$ & $19,04 \mathrm{a}$ & $67,08 \mathrm{~b}$ & $3,75 \mathrm{~b}$ & $0,36 \mathrm{~b}$ & $8,44 \mathrm{bc}$ & $42,25 \mathrm{bc}$ \\
Compost-aserrín & $\mathrm{T} 4$ & $13,66 \mathrm{c}$ & $38,00 \mathrm{c}$ & $3,83 \mathrm{~b}$ & $0,27 \mathrm{~cd}$ & $6,33 \mathrm{de}$ & $22,27 \mathrm{~d}$ \\
Compost-musgo & $\mathrm{T} 5$ & $19,12 \mathrm{a}$ & $91,66 \mathrm{a}$ & $4,91 \mathrm{a}$ & $0,44 \mathrm{a}$ & $14,22 \mathrm{a}$ & $56,66 \mathrm{a}$ \\
Estiércol de caballo-aserrín & $\mathrm{T} 6$ & $16,27 \mathrm{abc}$ & $23,33 \mathrm{~d}$ & $2,50 \mathrm{~d}$ & $0,28 \mathrm{c}$ & $8,22 \mathrm{bc}$ & $09,30 \mathrm{e}$ \\
Estiércol de caballo-musgo & $\mathrm{T} 7$ & $14,75 \mathrm{bc}$ & $62,41 \mathrm{~b}$ & $3,58 \mathrm{bc}$ & $0,33 \mathrm{~b}$ & $7,11 \mathrm{~cd}$ & $33,58 \mathrm{c}$ \\
\hline Cac
\end{tabular}

Cada par de tratamientos con la misma letra no son significativos al nivel de 0,05\% (Prueba de Rango Múltiple Duncan).

$\mathrm{Ap}=$ altura de planta, $\mathrm{N}^{\circ} \mathrm{h}=$ número de hojas por planta, $\mathrm{N}^{\circ} \mathrm{b}=$ número de broes por planta, $\mathrm{Dt}=$ diámetro de tallo por planta, $\mathrm{N}^{\circ} \mathrm{r}=$ número de raíces por planta, $\mathrm{N}^{\circ} \mathrm{f}=$ número de flores por planta.

Elresultado favorable obtenido se debióa la composición del sustrato (compost y musgo). El musgo por ser de la zona altoandina fría, al ser utilizado en condiciones de altas temperaturas y bajo la presencia de microorganismos se acelera el proceso de descomposición y mineralización; facilitando la disponibilidad de los nutrientes necesarios en el sustrato. Sin embargo, Valenzuela et al. (2003) reportó que cuando el sustrato está compuesto solo por compost el tamaño de la planta se ve afectado negativamente debido al $\mathrm{pH}$ alcalino que perjudica la disponibilidad de nutrientes. La presencia de sales en los insumos tierra de chacra, compost y estiércol de caballo provocaron desórdenes fisiológicos, desequilibrios iónicos, disminución en el potencial hídrico del medio nutritivo y restricción de la absorción de agua por las raíces. Las presencias de aserrín en estos tratamientos contribuyeron al incremento de la relación C/N de 80-150 (Stoffella \& Kahn, 2005), originando la competencia de los microorganismos descomponedores de la materia orgánica con la raíz por tomar el nitrógeno. Dicho resultado es corroborado por Gene (2010) quien determinó que el clavel es un cultivo susceptible a la deficiencia de nitrógeno, teniendo como resultado final un menor número de hojas.

\section{Fucsia (Fuchsia sp.)}

En la Tabla 4 se muestra los resultados de las variables evaluadas en la Fuchsia sp. por tratamiento, donde el T5 mostró la mejor respuesta en todas las variables evaluadas en comparación con los demás tratamientos. Arancibia (1993), en un ensayo que realizó con Fuchsia y Tagetes obtuvo un mejor crecimiento y desarrollo de los cultivos con el sustrato que estuvo compuesto por tierra de hoja más $10 \%$ de humus de lombriz. Los resultados obtenidos coinciden con Sarmiento (2011) quien reporto mejores resultados para el cultivo de maíz y acacia con el sustrato elaborado de la corteza de pino compostada; ello se debió, porque la materia orgánica al estar compostada disminuye la relación $\mathrm{C} / \mathrm{N}$, debido a la actividad de los microorganismos presentes en la compostación que van degradando los compuestos carbonatados como la lignina y se van transformando en compuestos más simples que pasan a ser constituyentes de las bacterias, y estas al morir quedan disponibles para ser tomadas por las raíces ya están compuestas de proteínas y enzimas que las planta pueden absorber.

El T5 llegó a producir 31,91 flores, resultado favorable en comparación con los demás tratamientos. Los T2, T3 y T7 llegaron a producir 9,58; 7,25 y 9,72 flores respectivamente, resultado inferior al T5. Sin embargo, los T1 y T6 no llegaron producir flores. Ello se debió a los insumos utilizados en los sustratos, cuyos valores de conductividad eléctrica de $3,63 \mathrm{dSm}^{-1}$ (tierra de chacra); $4,69 \mathrm{dSm}^{-1}$ (compost) y $20,70 \mathrm{dSm}^{-1}$ (estiércol de caballo) originan una situación crítica de sales solubles que incrementa la presión osmótica en la solución acuosa. Consecuencia de ello, la planta presenta desórdenes fisiológicos, desequilibrios iónicos y disminución del potencial hídrico de la solución acuosa; restringiéndose la absorción de agua y sales minerales por parte de la raíz (Mengel y Kirkby, 2004). También se observó una alta relación C/N 80-150 (Stoffella \& Kahn, 2005) por la presencia del aserrín que provoca deficiencia de nitrógeno en la solución nutritiva. Sin embargo, Ortega et al. (2010) encontró que el sustrato cuya mezcla es aserrín con lombricomposta tiene efectos similares a la turba en cuanto a altura, diámetro de tallo y mayor peso seco; esto puede deberse a que el aserrín que se utilizó en dicho ensayo fue compostado previamente, por lo que esta materia orgánica presentó una relación $\mathrm{C} / \mathrm{N}$ más baja.

Tabla 4. Resumen de las variables evaluadas en Fuchsia sp. en siete diferentes sustratos

\begin{tabular}{lcccccr}
\hline \multicolumn{1}{c}{ Tratamientos } & $\mathrm{T}$ & $\begin{array}{c}\mathrm{A} \mathrm{p} \\
(\mathrm{cm})\end{array}$ & $\begin{array}{c}\mathrm{N}^{\circ} \mathrm{h} \\
(\mathrm{und})\end{array}$ & $\begin{array}{c}\mathrm{D} \mathrm{t} \\
(\mathrm{cm})\end{array}$ & $\begin{array}{c}\mathrm{N}^{\circ} \mathrm{r} \\
(\mathrm{und})\end{array}$ & $\begin{array}{r}\mathrm{N}^{\circ} \mathrm{f} \\
(\mathrm{und})\end{array}$ \\
\hline Tierra de chacra- aserrín & $\mathrm{T} 1$ & $15,71 \mathrm{e}$ & $09,66 \mathrm{~d}$ & $0,34 \mathrm{~d}$ & $3,11 \mathrm{~d}$ & $0 \mathrm{c}$ \\
Tierra de chacra-compost & $\mathrm{T} 2$ & $62,02 \mathrm{a}$ & $18,08 \mathrm{ab}$ & $0,77 \mathrm{ab}$ & $6,11 \mathrm{abc}$ & $9,58 \mathrm{~b}$ \\
Tierra de chacra-musgo & $\mathrm{T} 3$ & $60,64 \mathrm{a}$ & $18,41 \mathrm{ab}$ & $0,72 \mathrm{~b}$ & $7,22 \mathrm{a}$ & $7,25 \mathrm{~b}$ \\
Compost-aserrín & T4 & $41,87 \mathrm{c}$ & $09,91 \mathrm{~d}$ & $0,44 \mathrm{c}$ & $4,11 \mathrm{~cd}$ & $0 \mathrm{c}$ \\
Compost-musgo & T5 & $60,90 \mathrm{a}$ & $19,58 \mathrm{a}$ & $0,79 \mathrm{a}$ & $6,66 \mathrm{ab}$ & $31,91 \mathrm{a}$ \\
Estiércol de caballo-aserrín & $\mathrm{T} 6$ & $24,33 \mathrm{~d}$ & $13,00 \mathrm{c}$ & $0,34 \mathrm{~d}$ & $3,11 \mathrm{~d}$ & $0 \mathrm{c}$ \\
Estiércol de caballo-musgo & T7 & $53,15 \mathrm{~b}$ & $15,83 \mathrm{~b}$ & $0,71 \mathrm{~b}$ & $5,11 \mathrm{bcd}$ & $9,72 \mathrm{~b}$ \\
\hline
\end{tabular}

Cada par de tratamientos con la misma letra no son significativos al nivel de $0.05 \%$ (Prueba de Rango Múltiple Duncan).

$\mathrm{Ap}=$ altura de planta, $\mathrm{N}{ }^{\circ} \mathrm{h}=$ número de hojas por planta, $\mathrm{Dt}=$ diámetro de tallo por planta, $\mathrm{N}^{\circ} \mathrm{r}=$ número de raíces por planta, $\mathrm{N}^{\circ} \mathrm{f}=$ número de flores por planta. 


\section{Conclusiones}

El mejor sustrato es la mezcla de compost-musgo, con dicha mezcla se obtendrá mejor altura, número de hojas, diámetro de tallo, número de raíces y flores por planta en las dos especies ornamentales Dianthus chinensis y Fuchsia sp. mientras que con mezclas para ambos cultivos los T1 (tierra de chacra-aserrín), T4 (compost-aserrín) y T6 (estiércol de caballo-aserrín) que presentaron como insumos tierra de chacra, compost y estiércol de caballo se tendrá menor rendimiento en las variables crecimiento y rendimiento, debido principalmente a la presencia de sales en concentraciones perjudiciales para el crecimiento y desarrollo del cultivo. Así mismo, la presencia de aserrín en los tratamientos mencionados, afectó la disponibilidad de nutrientes en la solución acuosa.

\section{Literatura citada}

Abad, M. 1993. Sustratos. Características y propiedades. pp. 47-62. In: Cultivos sin suelo. F. Cánovas y J.R. Díaz. (ed.). Instituto de Estudios Almerienses. FIAPA.

Abad, M. y Noguera, P. 2000. Los sustratos en los cultivos sin suelo. In: Urrestarazu, M. G. (ed.). Manual del Cultivo sin Suelo. Mundi-Prensa. Almería, España. 137-184p.

Abad, M.; Noguera, P.; Puchades, R.; Maquieira, A. and Noguera, V. 2005. Physico-chemical and chemical properties of some coconut coir dusts for use as a peat substitute for containerised ornamental plants. Biores. Technol. 82:241-245.

Ansorena, M.J. 1994. Sustratos. Propiedades y Caracterización. Mundi-Prensa. Madrid, España. 170 p.

Arancibia, E. 1993. Utilization of alternative growing media in replacement of compost. Disponible en: http://agris. fao.org/agris-search/search.do?recordID $=$ CL9500669

Armijos, M. y Barrezueta, S. 2016. Análisis de la cadena de comercialización de plantas ornamentales de los viveros en la ciudad de Machala, Ecuador, Revista Caribeña de Ciencias Sociales. Disponible en: http:// www.eumed.net/rev/caribe/2016/05/viveros.html http://hdl.handle.net/20.500.11763/CARIBE-2016-05viveros

Avenza. 2018. UF0019: Preparación del medio de cultivo.

Editorial ICEditorial. Disponible en: https://books. google.com.pe/books?id=oGtUDwAAQBAJ\& printsec $=$ frontcover $\& d q=$ inauthor: $\% 22 \% \mathrm{C} 3 \%$ 811 varo + Avenza $+\%$ C $3 \% 81$ lvarez $\% 22 \&$ hl $=$ es 419\&sa $=$ X\&ved $=0$ ahUKEwjZxZ_k6tbeAhUKvFkKH f8JBwsQ6AEIKzAB\#v=onepage \&q\&f=false

Barzegar, Z.; Mobli, M. and Abdossi, V. 2015. Comparison of different culture media on growth and yield of bell peppers (Capsicum annum). Nov. Appl Sci. 4(8): 900904.

Baudoin, W.; Nisen, A.; Grafiadellis, M.; Verlodt, H.; Jiménez, R.; De Villele, O. y Monteiro, A. 2002. El cultivo protegido en el clima mediterráneo. Medios y Técnicas de Producción. Suelo y Sustratos. FAO. Roma. 143-182p.

Burés, S. 1997. Sustratos. Ediciones Agrotécnicas. Madrid, España. 342 p.

Burés, S. 1998. Sustratos para la producción viverística. En: Ballester-Olmos, J.F. (Ed.). Producción de plantas ornamentales. 97-102p. Universidad politécnica de Valencia.

Cabrera, R.I. 1999. Propiedades, uso y manejo de sustratos de cultivo para la producción de plantas en macetas Revista Chapingo Serie Horticultura, 5 (1): 5-11.

Eltelégrafo. 2017. El uso de cuatro especies de plantas para los nacimientos está prohibido. Disponible en: https:// www.eltelegrafo.com.ec/noticias/sociedad/1/el-usode-cuatro-especies-de-plantas-para-los-nacimientosesta-prohibido

Fortis-Hernández, M.; Preciado-Rangel, P.; GarcíaHernández, J.; Navarro, A.; Antonio-González, J. y Omaña, J. 2012. Sustratos orgánicos en la producción de chile pimiento morrón. Revista mexicana de ciencias agrícolas, 3(6): 1203-1216. Disponible en: http://www. scielo.org.mx/pdf/remexca/v3n6/v3n6a11.pdf

Gayosso, S.; Borges, L.; Villanueva, E.; Estrada, M.A. y Garruña, R. 2016. Sustratos para producción de flores. Agrociencia, 50(5): 617- 631.

Gayosso-Rodríguez, S.; Villanueva-Couoh, E.; EstradaBotello, M. y Garruña, R. 2018. Caracterización físicoquímica de mezclas de residuos orgánicos utilizados como sustratos agrícolas. Bioagro, 30(3): 179-190. Disponible en: http://www.ucla.edu.ve/bioagro/ REV30(3)/2.\%20ms\%201754.pdf

Garcia, E. 2004. Modificaciones al sistema de clasificación climática de Köppen. Quinta edición, Instituto de Geografía -UNAM. Disponible en: Mexico http:// www.igeograf.unam.mx/sigg/utilidades/docs/pdfs/ publicaciones/geo_siglo21/serie_lib/modific_al_sis. pdf

Handreck, K.A. and Black, N.D. 2002. Growing Media for Ornamental Plants and Turf. UNSW Press.

Instituto Metropolitano de Planificación. 2012. Plan regional de desarrollo concertado de Lima. 441 p.

Iskander, C. R. 2002. Manejo de sustratos para la producción de plantas ornamentales en maceta. Department of Horticultural Sciences, Texas A\&M University.

Hartmann, T.H. y Kester, D.E. 1998. Propagación de Plantas; Principios y Prácticas. CECSA. 2da ed. México. 760p.

Huarcaya, P. 2014. Efecto de diferentes tipos de sustrato en la producción de semilla prebásica de papa (Solanum tuberosum La) en condiciones de Acobamba, Huancavelica. Tesis de Ingeniero Agrónomo, Universidad Nacional de Huancavellca, Acobamba, Huancavelica, Perú. 55p. 
Jardinería. 2016. Propiedades del estiércol de caballo. Disponible en: https://www.jardineriaon.com/ propiedades-del-estiercol-caballo.html

Jaulis, J. y Pacheco, A. 2015. Producción de marigold (Tagetes patula cv. Durango orange) en diferentes medios de crecimiento, bajo condiciones de vivero de la Universidad Nacional Agraria La Molina. Anales científicos, 76 (1): 38-43.

Juárez T.P.; Rubio A.; Narváez F. y Jiménez C. 2001. Comparación de ocho tratamientos para la elaboración de composta a base de aserrín y estiércol. In: Memorias del V Congreso Mexicano de Recursos Forestales. Universidad de Guadalajara, CUCBA. SOMEREFO. Guadalajara, Jalisco. 395-396p.

López-Baltazar, J.; Méndez-Matías, A.; Pliego-Marín, L.; Aragón-Robles, E. y Robles-Martínez, M. 2013. Evaluación agronómica de sustratos en plántulas de chile 'onza' (Capsicum annuum) en invernadero. Revista Mexicana de Ciencias Agrícolas, 6:1139-1150.

Mascarini, L.; Lorenzo, G.; Svartz,H.; Pesenti, S. y Amado, S. 2012. Tamaño de contenedor y tipo de sustrato afectan la eficiencia en el uso del agua en Gerbera jamesonii para flor cortada. Rev. Bras. Hort. Ornam., 18: 71-77.

Mengel, K.; Kirkby, EA. 2004. Principios de nutrición vegetal. Annals of Botany, 93 (4): 479-480. Disponible en: https:/www.ncbi.nlm.nih.gov/pmc/articles/ PMC4242342/

Morales M.E.R. y Casanova, L. 2015. Mezclas de sustratos orgánicos e inorgánicos, tamaño de partícula y proporción. Agron. Mesoam., 26: 365-372.

Gene B. J. 2010. Characterization of nutrient disorder of floriculture species. Thesis Master of Science. North Carolina State University. USA. 216p.

Oberpaur, C.; Puebla, V.; Vaccarezza F. and Arévalo, M. 2010. Preliminary substrate mixtures including peat moss (Sphagnum magellanicum) for vegetable crop nurseries. Cien. Inv. Agr. 37(1):123-132.

Ortega, M. L.; Sánchez, O.; Ocampo, M.; Sandoval, C.; Salcido, R. y Manzo, R. 2010. Efecto de diferentes sustratos en crecimiento y rendimiento de tomate (Lycopersicum esculentum Mill.) bajo condiciones de invernadero. Ra Ximhai. 6(3): 339-346.

Oviedo-Ocaña, R.; Marmolejo-Rebellon, L. y TorresLozada, P. 2012. Perspectivas de aplicación del compostaje de biorresiduos provenientes de residuos sólidos municipales. un enfoque desde lo global a lo local. Revista Ingenierías Universidad de Medellín, 11 (20): 67-76. Disponible en: http://www.scielo.org.co/ pdf/rium/v11n20/v11n20a06.pdf

Palacios, J.A. 2007. Manual de manejo de viveros. Universidad nacional Agraria la Molina.

Pastor, S. 2000. Utilización de sustratos en viveros. Terra Latinoam, 17: 231-235.

Pérez, J.; López, L. y Romero, R. 2016. “Automatizacion de invernadero para cultivos hidropónicos en El Salvador. Trabajo de Investigación, Escuela Especializada en Ingeniería Mecatrónica Escuela de Ingeniería en Mecatrónica. Santa Tecla, La Libertad, El Salvador. Disponible en: http://www.redicces.org.sv/jspui/ bitstream/10972/2843/1/Automatizaci\%C3\%B3n\%20 de $\% 20$ Invernadero $\% 20$ para $\% 20$ Cultivos $\% 20$ Hidrop\%C3\%B3nicos\%20en\%20El\%20Salvador.pdf

Pineda-Pineda, J.; Sánchez del Castillo, F.; RamírezArias, A.; Castillo-González, A.; Valdés-Aguilar, L. y Moreno-Pérez, E. 2012. Aserrín de pino como sustrato hidropónico. I: Variación en características físicas durante cinco ciclos de cultivo. Revista Chapingo. Serie horticultura, 18(1): 95-111.

Quintero, C.; González, M. y Guzmán, P. 2011. Sustratos para cultivos hortícolas y flores de corte. In: Flórez R., V. J. (ed). Sustratos, Manejo del Clima, Automatización y Control en Sistemas de Cultivo sin Suelo. Universidad Nacional de Bogotá. Colombia. 79-108p.

RPP. 2017. Lima tiene un déficit de 56 millones de metros cuadrados en áreas verdes. Disponible en: https:// rpp.pe/data/lima-tiene-un-deficit-de-61-millones-demetros-cuadrados-en-areas-verdes-noticia-1021931

Sakata Seed Sudamerica LTDA. 2011. Dianthus F1 Diamond. Disponible en: http://www.sakata.com/index.ph $\mathrm{p}$ ? action $=$ catalogo $\&$ cultura $=3 \&$ producto $=4 \&$ lenguaje $=\mathrm{pt}$.

Sarmiento, O.M. 2011. Alternativas de compostaje de aserrín de pino Caribe (Pinus caribaea) en la industria maderera Refocosta S.A., municipio de Villanueva, Casanare, Colombia. En: Revista de Investigación Agraria y Ambiental (RIAA), 2(2). Ed. Reinaldo Giraldo Díaz.

Stoffella, P. \& Kahn, B. 2005. Utilización de compost en los sistemas de cultivo horticola. Ediciones MundiPrensa Madrid Barcelona, México, 379p. Disponible en: http://redbiblio.unne.edu.ar/pdf/0603-001931_I. pdf

Tortarolo, M.; Pereda, M.; Palma, M. y Arrigo, N. 2008. Influencia de la inoculación de microorganismos sobre la temperatura en el proceso de compostaje. Ciencia del suelo, 26(1): 41-50. Disponible en: http://www. scielo.org.ar/pdf/cds/v26n1/v26n1a05.pdf

Urrestarazu, M. 2015. Manual Práctico del Cultivo sin Suelo e Hidroponía. Mundi-Prensa. Madrid, España. $278 \mathrm{p}$.

Valenzuela, R.; Gallardo, C.S.; Carponi, M.S.; Aranguren, M.E.; Tabares, H.R. y Barrera, M.C. 2014. Manejo de las propiedades físicas en sustratos regionales para el cultivo de plantas en contenedores. Ciencia, Docencia y Tecnología, 4(4): 1-19.

Valenzuela, O.; Nicolau, F.; Rode, M.; Schlund, H. y Gallardo, C. 2003. Respuesta de Tagetes patula a sustratos formulados con compost y perlita. En: Revista científica agropecuaria, 7(1): 57-61. Facultad de ciencias agropecuarias UNER. 
Vargas, J.M.; Castillo, A.M.; Pineda, J.; Ramírez, J.A. y Avitia, E. 2014. Extracción nutrimental de jitomate (Solanum lycopersicum L.) en mezclas de tezontle con aserrín nuevo y reciclado. Revista Chapingo Serie Horticultura, 20(1): 71-88.

VIFINEX. 2002. Producción de sustratos para viveros. Disponible en: http://www.cropprotection.es/ documentos/Compostaje/Sustratos-para-Viveros.pdf

Yagodín, B. 1986. “Agroquímica”. Editorial Mir Moscú. URSS.

Zurbrügg, et al. 2005. Descentralised composting in Bangladesh a win - win situation for all stakeholders.

Resources Conservation \& Recycling, 43: 281-292. 